\title{
Anesthesia Management for Carotid Endarterectomy: Review Article
}

\section{(1) Fulya Yılmaz}

Health Science University İzmir Bozyaka Training and Research Hospital, Clinic of Anaesthesiology and Reanimation, İzmir, Turkey

\begin{abstract}
Atherosclerotic carotid artery disease is responsible from $20 \%$ to $25 \%$ of ischemic stroke events. Open carotid surgery and stent insertion are two main types of treatment procedures. Carotid endarterectomy (CEA) can be performed under general anaesthesia, regional anaesthesia (interscalen block, cervical plexus block either by landmark technique or by US guidance), combination of general and regional anaesthesia, regional anaesthesia with combination of periferic block. The aim of all anesthesic techniques is to prevent pain during the three painful periods (1. Skin incision, 2. Insertion of a retromandibular retractor, 3. Perivascular preparation) during CEA). All techniques have their own advantages and disadvantages. But there is no certain data about which one is superior to other. There
\end{abstract}

\section{Introduction}

Atherosclerotic carotid artery disease, which leads to cerebrovasculer diseases by embolization of atheromatous material or thrombotic occlusion, responsible from $20 \%$ is no consensus on anaesthetic choice for CEA. At present regional anaesthesia versus general anaesthesia for patients undergoing CEA is still debate topic. Improvements in medical theraphy, use of cerebral monitoring, better timing for surgery after ischemic events, better surgical techniques, increased use of ultrasound for regional anaesthesia improve procedural outcomes. Despite these improvements, anaesthesia management is still deciding according to surgeon and anaesthesiologist preferences, the patient's satisfaction and the conditions in the hospitals where we work.

Keywords: Carotid endarterectomy, anaesthesia techniques, general anaesthesia, cervical plexus block, local anaesthesia

\footnotetext{
Address for Correspondence: Fulya Y1lmaz, Health Science University İzmir Bozyaka Training and Research Hospital Department of Anaesthesiology and Reanimation, İzmir, Turkey

Phone: +90 5335110505 e-mail: fulya.dr@gmail.com ORCID ID: orcid.org/0000-0002-6901-7404

Received: 23.02.2019 Accepted: 22.05.2019
}

Cite this article as: Y1lmaz F. Anesthesia Management for Carotid Endarterectomy: Review article. EJCM 2019;7(2):50-59.

DOI: 10.32596/ejcm.galenos.2019.00010

${ }^{\circ}$ Copyright 2019 by Heart and Health Foundation of Turkey (TÜSAV) / E Journal of Cardiovascular Medicine published by Galenos Publishing House. 
since 1970, CEA accepted as the best treatment option in patients who has high grade carotid artery stenosis ${ }^{(7)}$. While CEA is accepted as "gold standart" in symptomatic patients with stenosis $>70 \%$; there is still a controversy in management of asymptomatic patients ${ }^{(2,3,5,8,9)}$. Stent insertion for carotid stenosis was first reported by Kerber et al. in 1980, approximately 30 years after the $\mathrm{CEA}^{(6)}$. Two main types of techniques are used in CEA: ventrojugular approach (the standart surgical technique) and retrojugular approach (improve patient tolerance and facilitate the surgical procedure $)^{(5)}$. CEA is a preventive surgery and useful in symptomatic patients if the operation can be done within two weeks. On the other hand, patients undergoing CEA have so many comorbidities and prone to intraoperative hemodynamic fluctuations ${ }^{(6,10,11-13)}$. Although anaesthesiologists have a very limited time to optimize the patient, they should also select the most appropriate method for anaesthesia management ${ }^{(10,14)}$.

CEA can be performed under general anaesthesia, regional anaesthesia [interscalen block, cervical plexus block either by landmark technique or by ultrasound (US) guidance], combination of general and regional anaesthesia, regional anaesthesia with combination of periferic block ${ }^{(8,10)}$. The aim of all anesthesic techniques is to prevent pain during the three painful periodss (1. Skin incision, 2. Insertion of a retromandibular retractor, 3. Perivascular preparation) during $\mathrm{CEA}^{(15)}$. All techniques have their own advantages and disadvantages. But there is no certain data about which one is superior to other ${ }^{(8-10,16-18)}$. Radak et al. conducted a study to answer the question "Should the guidelines now be updated for CEA?". They reported that improves after CEA procedures can be explained by improvements in medical therapy, more appropriate timing of CEA, the use of local anaesthesia and the se of preoperative cerebral monitoring and as well as improves in surgical techniques ${ }^{(6)}$.

The General versus Local Anaesthesia which is the only large randomized control study comparing general anaesthesia (GA) and regional anaesthesia (RA) (Landmark guided superficial cervical plexus block or deep cervical plexus block) for CEA, conclude that there is no difference in myocardial infarction (MI), death and stroke when comparing GA with RA. On the contrary shunt requirement was $50 \%$ in GA group, $14 \%$ in RA group ${ }^{(19)}$. Moulakakis et al. ${ }^{(20)}$ advocate to perform a study, compared CEA under RA without shunting, even if patients manifest neurological symptoms, compared with CEA under GA without shunting, answer the question: "The role of shunting in preventation of stroke."

NSQIP (National Surgical Quality Improvement Program) study compared 30-day postoperative MI, stroke, and mortality between CEA under RA or local anaesthesia (LA) and CEA under GA. NSQIP study analysis suggests that CEA under GA has an increased risk of postoperative MI. They prefer patients to undergo CEA either by RA or LA, who have risk for postoperative $\mathrm{MI}^{(4)}$.

Malik et al. demonstrated that the use of RA versus GA in CEA is associated with decreased risk of postoperative pneumonia and reduced need for perioperative blood transfusions ${ }^{(18)}$. Markovic et al. reported that type of anaesthesia does not affect the outcome of surgical treatment of CEA but regional techniques are related with fewer respiratory complications, later requirement for first postoperative analgesic and awake patient after surgery (who can continue oral theraphy earlier) ${ }^{(21)}$. Lobo et al. reviewed their 10 year practice of GA and locoregional anaesthesia for CEA. They showed that the number of studies about impact of anaesthesia type (evaluating delirium, cognitive impairment and quality of life after surgery) is very small. This indicates a need for randomized studies ${ }^{(22)}$.

Cross-clemp intolerance (CCI) is a rare event during CEA. Hypertension and symptomatic carotid stenosis were independent and significant risk factors for CCI. Recently, in selective patients, carotis artery stending (CAS) has been used as alternative treatment for CEA who developed CCI during CEA. If patient has risk factors for CCI, GA is more suitable option as anaesthesic method because of risk for shunting or conversion to $\mathrm{CAS}^{(23)}$. On the other hand, Dellaretti et 
al. demonstrated that locoregional anaesthesia is a safe method for identifying patients with CCI and the mean degree of contralateral carotis stenosis was associated with $\mathrm{CCI}^{(24)}$. Several CREST (Carotid Revascularization Endarterectomy versus Stenting Trial) showed that CAS was associated with a significantly higher risk of stroke, CEA is associated with higher incidence of MI. In other CREST study performed by Hye et al. reported that risk of periprocedural MI was similar in patients undergoing CEA under RA and those undergoing CAS. But the risk was doubled in CEA under GA compared to the patients undergoing $\mathrm{CAS}^{(25)}$. Hoefer et al. assessed early sympathetic activity during CEA in ultrason guidedintermediate cervical plexus block with GA. Although, United States-Information and Communications Policy Bureau (US-ICPB) is a feasible, effective and safe form of RA for CEA; it induces temporary intraoperative hypertension and increase the stress hormone levels ${ }^{(26)}$.

\section{Anaesthesia methods for carotid endarterectomy:}

1. General anaesthesia: GA is still the most preferred anaesthetic technique for $\mathrm{CEA}^{(1,27)}$.

\section{Advantages of GA are:}

- Control of ventilation (Tight control of arterial carbon dioxide concentration $)^{(1,3,9,14,28)}$

- Cerebral protection afforded by volatile anaethesia (especially during the cross-clamp) $)^{(1,2,9,10,25,29)}$

- Airway security (Anaesthetist preference) $)^{(1,7,9,10,28)}$

- Excellent and comfortable operation conditions (especially in patients with high carotid bifurcation) $(3,7,10,25,29)$

- Prevent myocardial ischemia ${ }^{(10)}$

- Less stressful than a regional procedure ${ }^{(3,25)}$

- Prevent patients from coughing and straining ${ }^{(10)}$

- Possibility of inducing hypothermia if required ${ }^{(9)}$

\section{Disadvantages of GA are:}

- GA necessitates shunt insertion more commonly than RA techniques ${ }^{(3,7,30)}$

- Requires brain monitoring (stump pressure, somatosensory evoked potential, transcranial Doppler, EEG and near-infrared spectroscopy, juguler venous oxygen,cerebral oximetry $)^{(3,7,9,14,30)}$. These methods shows poor sensitivity and specificity according to awake patient, in terms of requirement for shunt placement ${ }^{(9,11)}$.

- Anaesthetic-induced circulatuar depression is associated with labile blood pressure ${ }^{(3)}$ and necessitates greater vasopressor support (to ensure adequate cerebral perfusion pressure during carotid cross-clemping) ${ }^{(3,7)}$

- Higher incidence in postoperative neurocognitive dysfunction $^{(7,27,28)}$

$\Rightarrow$ Only general anaesthesia: General anaesthesia can be performed by intubation (preferred) or by laryngeal mask airway (in difficult airway circumstance) for $\mathrm{CEA}^{(7,10)}$. General anaesthesia can be managed using inhaled or total intravenous agents Cerebral metabolic rate reductions are similar with propofol and halogenated volatile agents but cerebral blood flow is lower with propofol than halogenated volatile agent ${ }^{(27)}$.

Kuzkov et al. reported that sevoflurane (induction and maintenance of anaesthesia with sevoflurane) is better than propofol (Total intravenous anaesthesia with propofol) to improve early postoperative cognitive dysfunction ${ }^{(31)}$. Wang et al. results offers low-dose sevoflurane with propofol in patients undergoing CEA reduces the incidence of myocardial injury in symptomatic patients after $\mathrm{CEA}^{(32)}$.

Tsujikawa et al. reported that low-dose dexmedetomidine improves hemodynamic stability during the emergence and the recovery phases of general anaesthesia in patients undergoing CEA, in their randomized double-blind placebocontrol trial ${ }^{(29)}$.

$\Rightarrow$ General anaesthesia with superficial cervical plexus block: Addition of superficial-CPB to general anaesthesia provides lower VAS scores at discharge from the postoperative care unit ${ }^{(5)}$.

$\Rightarrow$ Combined general anaesthesia with regional anaesthesia: Recent studies reported that the quality of block should be evaluated according to the "patient 
satisfaction". So new trend is combination of RA with $\mathrm{GA}^{(9)}$.

$\Rightarrow$ General anaesthesia with cooperative patient: It is like "wake-up" test performed during neurosurgical surgeries ${ }^{(1,9)}$. Anaesthesia is induced and maintained with propofol and remifentanil. Before clamping, doses of drugs are reduced until patient obey the verbal comments $^{(1)}$. It was first used by Baldinelli during $\mathrm{CEA}^{(9)}$. The first pilot study, performed by Luchetti, reported that it guaranteed more stable hemodynamic conditions, better patient comfort and more effective analgesia as compared to superficial cervical plexus block alone during $\mathrm{CEA}^{(33)}$. Then Bevilacqua defined a new technique: "Cooperative Patient General Anaesthesia" (Co.PA.Ge.A.), which is similar to Luchetti technique. In this technique, LA is injected to the anterior borer of sternocleidomastoid muscle, instead of superficial cervical plexus block. In these three studies remifentanil was used as opioid in general anaesthesia application and demonstrated as safe and with low rate of conversion to $\mathrm{GA}^{(9)}$.

Regional anaesthesia: "Right dose of the right drug placed in the right place" is a sentence that describes the "gold standart" in every nerve block ${ }^{(1,9)}$.

\section{Advantages of regional anaesthesia are:}

- Awake patient is the gold standart cerebral function monitoring ${ }^{(1,3,9,11,12,14,17,28,34)}$

- Reduce cardiac and respiratory related morbidity ${ }^{(1,3,30,34)}$,

- Lower shunt insertion rate rat, $^{(1,9,16,25,30)}$,

- Shorten hospital stay ${ }^{(1,3,14,17,25,27,34)}$

- Lower $\cos ^{(1,3,25,27)}$,

- Improves outcomes after surgery (better postoperative analgesia, reduce blood loss, lower risk of tromboembolic events) ${ }^{(1,14,25)}$,

- Safer ${ }^{(1)}$,

- Safe method to identify the patients at risk for crossclamp intolerance ${ }^{(1,3,12)}$,

- Hemodynamic stability ${ }^{(9,10,12,17,25,28)}$,
- Preserved cerebral autoregulation ${ }^{(3,10,28,30)}$,

- Reduce vasopressor requirement ${ }^{(28)}$,

- Reduces operative time ${ }^{(25)}$,

- Disadvantages of regional anaesthesia are:

- Needs patient collaboration ${ }^{(3,7,28)}$,

- Procedural complications (diaphragmatic and vocal cord paralysis, neural injuries, epidural, subaracnoid or intravascular injection of local anaesthetics) ${ }^{(7)}$,

- Urgent conversion of regional anaesthesia into general anaesthesia ${ }^{(7,9,35)}$,

- Anxiety during the operation ${ }^{(9,35)}$.

$\Rightarrow$ Cervical epidural block: For CEA an epidural catheter is sited at C6-C7 level and a dilue LA solution is injected (exp: $0.25 \%$ bupivacaine) ${ }^{(14)}$. It provides suitable operation conditions but associated with major anesthetic complications (dural tap, epidural hematoma, direct spinal cord damage $)^{(10,14)}$. But conversion to general anaesthesia is higher because of higher techniqual failure rate and higher rate of life-threatening complications ${ }^{(5,14,36,37)}$. Hakl et al. compared cervical epidural block and cervical plexus block (superficial and deep blocks) for failure rate and the frequency of anaesthesia related complications. Although both methods were acceptable for CEA, anaesthesia related complications are lower with $\mathrm{CPB}$. Failure rates were similar between the groups ${ }^{(37)}$.

$\Rightarrow$ Posterior cervical paravertebral block: Single injection, at $\mathrm{C} 4$ level, using nerve stimulator for blocking C2-4 dermatomes is another anaesthetic technique for CEA. By this technique, accidental vascular injury or injection is low ${ }^{(14)}$.

Cervical plexus block (CPB): $\mathrm{CPB}$ is commonly accepted RA technique for $\mathrm{CEA}^{(16)}$. Cervical plexus formed by anterior rami of four upper servical spinal nerves. It has two branches. 1. Superficial branches: provides cutaneous innervations to the head and anterolateral neck; 2. Deep branches: innervates muscles of the anterior neck, the anterior and middle scalen and the diaphragm ${ }^{(5,10,14)}$. Carotid surgery requires blockade of $\mathrm{C} 2-\mathrm{C} 3-\mathrm{C} 4$ cervical nerves ${ }^{(5,11,38)}$. Cervical fascia 
classified as superficial and deep fascia. Deep cervical fascia has three layers: Superficial layer (investing layer), middle layer and deep layer ${ }^{(39)}$. Figure 1 is showing the sides of injection for $\mathrm{CPBS}^{(34)}$. Regional anaesthesia can be performed by landmark technique or Ultrasound (US) guidance $^{(1)}$. There is a difficulty in classifiying the CPBs when landmark technique is used. Because exact location of the needle cannot known. If needle perforates the investing layer of the neck, this block should be termed as "intermediate block" instead of "superficial block". CPBs can be performed more thoroughly and safely by US-guidance ${ }^{(39)}$. US guidance during performing the block, permits direct visualization of nerve and other structures ${ }^{(1,9,17,38,39)}$, observation of the needle and LA spreading during injection ${ }^{(9,17,38,39)}$, increases the efficiency (faster onset and longer duration) and success rate, lower puncture related complications (avoidance of accidental vascular puncture), reduces LA dose (prevents tissue swallowing $)^{(3,7,9-11,15,17,38-41)}$. Disadvantages of CPB are palsy of the vagus nerve (which causes hoarseness, difficulty in swallowing, respiratory distress and are not clinically significant), conversion to general anaethesia because of block related complications or inadequate analgesia ${ }^{(1)}$. One of the major complication of RA is the need for urgent convertion to GA especially with cervical epidural block and deep servical block ${ }^{(1,3,8,10)}$. Indications for conversion from RA to GA are both surgery (loss of consciousness at carotid clamping, shunt related complications) and anaesthesia (block failure, pain and restlessness, claustrophobia, systemic toxicity of LA, airway obstruction, accidental subarachnoid injection, diaphragmatic or vocal cord paralysis causing respiratory distress) related ${ }^{(8)}$. Pandit et al conducted a study to assess the complication rates associated with superficial (or intermediate) and deep (or combined deep plus superficial/intermediate) ${ }^{(34)}$. They conclude that superficial/intermediate block is safer than any method that employs a deep injection. The main reason to convert GA with deep cervical plexus block (DCPB)/ deep combined CPB is direct complication of the block. Blocks combined with SCPB provides better analgesia. Pasin et al. reported that RA is the first-choice in their center ${ }^{(8)}$. In their practice, CEA under RA is safe and associated with very low rate of conversion to GA. The exact concentration and volume of LA for nerve blocks is still debated ${ }^{(9)}$. The block that was performed with lower dose LA under US-guidance is effective as that LA has to surround the entire nerve, "Doughnut sign", for

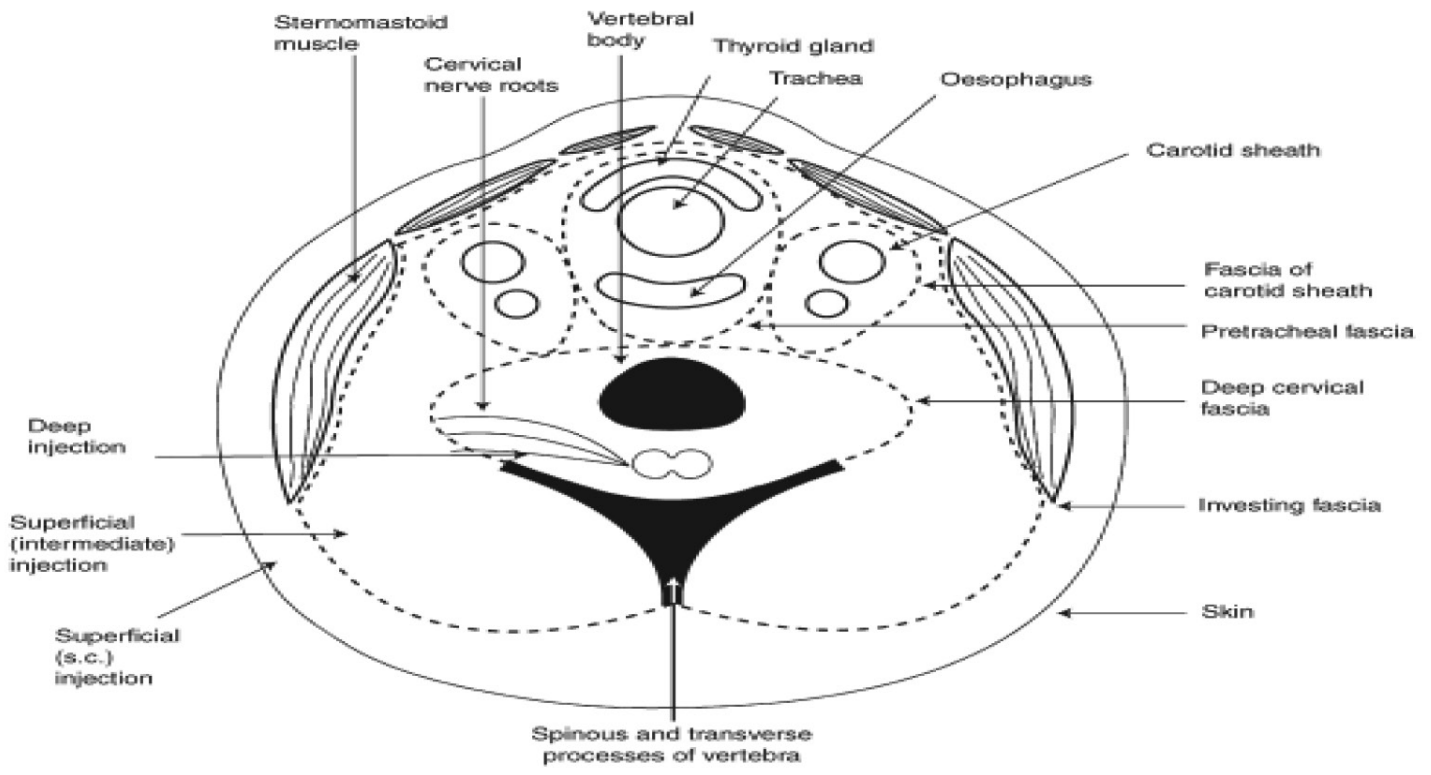

Figure 1. Cross-section of the neck at the C4 level, showing the sites of the injection of the deep, intermediate and superficial blocks 
successful block ${ }^{(9)}$. Clinicians may prefer one block to another but there is no consensus which one is the best ${ }^{(34)}$.

a. Superficial cervical plexus block (SCPB): Local anaesthetic is administered to posterior border of sternocleidomastoid muscle, superficial to the investing layer of deep cervical fascia ${ }^{(1,10,14,34,41,42)}$. It is a simple subcutaneous injection with an easy technique as described by Murphy and Scott ${ }^{(9,34,35,41)}$. SCPB can be performed under traditional or US-guidance technique. Nevertheless, the success rate of the techniques, onset time and total anaesthesia related times are similar ${ }^{(9,14)}$. The advantages US-guided technique over landmark technique are visualize the spread of LA and avoid accidental puncture of structures ${ }^{(14)}$. While large volumes $(20-30 \mathrm{~mL})$ of LA are used for SCPB by landmark technique; lower volumes (10-15 mL) of LA are used for SCPB by US-guidance ${ }^{(26)}$. It only provides analgesia, not paralyze the muscles ${ }^{(9,10,14)}$. According to Hariharan et al. experience, when SCPB is used alone, there is an increased need for supplementation of LA infiltration, especially during the dissection of the distal portion of $\mathrm{ICA}^{(43)}$.

SCPB is associated with lower complication rates ${ }^{(10)}$. Complications related with SCPB are hematoma, infection, local anesthetic toxicity and inadvertent intravascular injection $^{(1)}$.

b. Intermediate cervical plexus block (ICPB): In 2003 Pandit et al. in 2004, Telford and Stoneham defined "intermediate-CPB" as the injection of LA between the superficial and deep cervical fascia ${ }^{(9,14,38,39,42,44)}$. Local anaesthetic is administered under the investing layer of the deep fascia and superficial to the prevertebral fascia ${ }^{(1,10,34)}$. ICPB can be performed by landmark (loss of resistance or pop technique) or US-guided technique ${ }^{(38,42)}$. Choquet first described US-guided ICPB( ${ }^{(9)}$. US-guided ICPB can be performed anterior or posterior approach. Up to date, only one study described the technique of US-ICPB for CEA by using anterior approach. Authors reported that anterior approach is easy to perform than posterior approach $^{(38,39,42)}$. Complications of ICPB are similar with $\mathrm{SCPB}^{(1,42)}$. The authors showed that the deep cervical fascia is permeable ${ }^{(9,42)}$. It means that ICPB is safer than deep- $\mathrm{CPB}^{(9,42)}$, paralyzes the muscles like deep cervical plexus block ${ }^{(1,9,14,42)}$ but it doesn't associated with serious complications like deep cervical plexus $\operatorname{block}^{(9,10,14,42)}$. Theoretically, expectation from ICPB is to reduce the complications related with $\mathrm{DCPB}^{(14,38)}$.

Leblanc et al. reported that ICPB by US-guidance was safe (even in patients receiving antitrombotic agents), simple to perform, provide effective anaesthesia (with real-time neurological assessment and adequate haemodynamic stability) for CEA ${ }^{(12)}$.

Barone et al. reported that ICPB by landmark technique is feasible, effective and safe for CEA with low perioperative and postoperative complication rates ${ }^{(44)}$.

c. Deep cervical plexus block (DCPB): Local anaesthetic is administered under deep cervical fascia to cervical transverse process ${ }^{(1,14)}$ either by one single injection or multiple injection (three separate injections) $(10,14,16,34,42)$. The number of injections does not influence LA absorption as reflected by the Cmax and Tmax. Single injection technique can cause less pain during block and may be associated less systemic absorbtion of $\mathrm{LA}^{(45)}$; but there is no relationship between the number of injections and overall block efficacy ${ }^{(14)}$. DCPB can be performed by landmark or US-guided technique ${ }^{(9)}$. There are two approach for US guided technique: vertebral artery loop which can be easily identified by US, represents an accurate landmark for $\mathrm{C} 2$ transvers process ${ }^{(9,14)}$ or the longus capitis can be identified as a landmark ${ }^{(9)}$. It provides exact analgesia but it is technically difficult and associated with serious complications (epidural, subarachnoid, subdural or vertebral arter injection, seizures, recurrent laryngeal nerve palsy, phrenic nerve palsy, large neck hematoma, Horner's syndrome, higher convertion rate to $\mathrm{GA}^{(1,3,5,9,10,14,17,44)}$. By the introduction of US-guidance technique, the DCPB has become a relatively safe and simple procedure ${ }^{(42)}$.

While performing a deep CPB increasing number of injection from 1 to 3 , increasing the injected volume from from 10 to $40 \mathrm{~mL}$, observing a paresthesia, using 
neurostimulation or US guidance would not confor any clinically advantage over superficial CPB alone ${ }^{(5)}$.

C1. Continous deep cervical plexus block: This block is considered in high risk cases in which the procedure is expected to prolong ${ }^{(14)}$.

$\Rightarrow$ Combined block: Combined blocks appears as equally efficacious as $\mathrm{SCPB}^{(14)}$.

a. Superficial and deep cervical block: If locoregional anaethesia is choiced for CEA, combined superficial and deep CPB is preferred ${ }^{(44)}$. Although some authors demonstrated that combined superficial and deep $\mathrm{CPB}$ is more effective in producing muscle relaxation; others demonstrated that CEA can be successfully performed under S-CPB alone or combined $\operatorname{block}^{(9,14)}$. Muscle relaxation with DCPB has not been shown to be important clinically ${ }^{(42)}$. Kavakl1 et al. compared combined block (US-guided superficial and deep CPB) with USICPB for $\mathrm{CEA}^{(38)}$. Based on their result, combined block requires less analgesic requirement, provides lower VAS scores and higher patient satisfaction than US-ICPB.

b. Intermediate and deep cervical block: Another form of combined block ${ }^{(14,34)}$.

c. US-guided ICPB combined with carotid sheath infiltration: Madro et al. compare infiltration anaesthesia with US-guided ICPB combined with carotid sheath infiltration for CEA. They reported that combined block improves patient and surgeon comfort, safer, relatively simple, easy to master, requires little time to perform ${ }^{(36)}$.

$\Rightarrow$ US-guided carotid sheat block: Injection of LA to the carotid artery by US-guidance, the place of the operation, called as carotid sheat block instead of cervical plexus block by Casutt et al ${ }^{(11,17,41)}$. But Martusevicius et al. named the block as "locoregional anaesthesia" and Rössel et al. named the block as "perivascular regional anaesthesia". All authors reported that US-guided carotid sheat block can be performed rapidly, sufficient for surgery, lower supplemental LA during surgery and an alternative approach with lower complication rate ${ }^{(9,11,17)}$.
$\Rightarrow$ US-guided high interscalene plexus block: High-resolution US-guided (17.5-MHz device) high interscalene plexus block for CEA was first reported by Roessel et al. Although they reported that they were not encountered with complications, the small study group $(n=14)$ does not allow meaningful conclusion regarding safety of the procedure ${ }^{(46)}$.

$\Rightarrow$ Regional anaesthesia with combination of peripheric block: Performing peripheral blocks with US-guidance reduce incidence of LA toxicity compared to peripheral blocks that were performed without USguidance $^{(38)}$. Combining S-CPB with fascial nerve block may reduce the pain associated with prolonged use of retractor at the cephalic end of the incision ${ }^{(9)}$. Seidel et al. combined US-guided ICPB with new subplatsymal injection technique for peripheral fascial nerve block ${ }^{(15)}$. They reported that US-guided block is feasible, associated with high success rates, especially during initial skin incision. In another study, Seidel et al. combined USguided ICPB with perivascular LA infiltration; but they reported no clinical benefits of an additional periarterial LA infiltration ${ }^{(40)}$. Blocking mandibular division of trigeminal nerve by intraoral approach, alleviate the submandibular and dental pain which does not respond to administration of LA. It may be useful in patients with high carotid bifurcation or where atheromatous plaque extends cranially in the internal carotid artery ${ }^{(14,47)}$. Ozturk et al. compared the effect of combining CPB (US-guided SPCB and DCPB) with mandibular block for CEA. They reported that combining $\mathrm{CPB}$ with mandibular block provides better intraoperative pain control (lower intraoperative VAS scores, reduces intraoperative supplemental LA needs and intravenous analgesic requirement) and greater patient satisfaction than cervical plexus alone ${ }^{(47)}$.

$\Rightarrow$ Awake test under regional anaesthesia with sedation: Anxiety, uncomfortable neck position, traction pain are the problems that we encounter when we use RA alone for CEA ${ }^{(28,47)}$. Sedation can be administered as supplementtoregional blocks to overcame these limitations. Sedative agents have been used are: opioids (remifentanil, 
fentanyl), alfa-2-agonists (clonidine, dexmeditomidine), propofol $^{(3,5,14)}$, midazolam ${ }^{(5)}$, historically, butyrophenones (droperidol, haloperidol) $^{(3,14)}$ or with their combinations (midazolam-fentanyl) $)^{(5)}$. "Awake test" is the most reliable method for detecting hypoperfusion ${ }^{(28)}$. Studies in literature that compare RA with sedation with dexmedetomidine versus conventional sedation (fentanyl, remifentanyl or midazolam), prefers dexmedetomidine as sedative agent for CEA with RA. Because dexmedetomidine is a selective alpha-2 adrenoreceptor agonist with sedative, analgesic and anesthetic sparing effects. It does not suppress the respiratory function, maintains hemodynamic stability and patients can be awakened easily after cessation of its use $^{(14,28,29)}$.

2. Local anaesthesia: Local anaesthesia can be performed by two technique: Simple layer by layer to approach or four step approach (intradermal injection parallel to the anterior border of sternocleidomastoid muscle, subplatysmal injection, identification and individual infiltration of the cervical nerves and topical carotid anaesthesia) $)^{(5,14)}$.

Bhattathiri et al. studied effect of awake CEA under local anaesthesia with sedation on peri-operative blood pressure $^{(13)}$. Their study showed that local anaesthesia maintains a stable blood pressure during and immediately after CEA.

\section{Choice of local anaesthetic and adjuvants}

Other issue is LA choice for RA. Lidocaine, mepivacaine, bupivacaine, ropivacaine, levobupivacaine are suitable agents for $\mathrm{RA}^{(1,5,48)}$. If a mixture of $\mathrm{LA}$ is used, the total amount should be carefully predetermined as their toxicity is additive. Where bupivacaine offers the longest time, the mepivacaine the shortest time for first analgesic requirement ${ }^{(5)}$.

Cristallı et al. used ropivacaine versus levobupivacaine for cervical plexus block (deep and superficial block by anatomic landmark) to compare arterial blood pressure and incidence of hypotension. That reported ropivacaine as a first drug choice for $\mathrm{CPB}^{(48)}$.
Adjuvants may be added to LA to enhance the block efficiency $^{(9)}$. Epinephrine can be added to LA solution ${ }^{(1,5)}$. While epinephrine $1 / 200,000(5 \mathrm{mcg} / \mathrm{mL})$ increase the heart rate $15 \%$, epinephrine 1/300,000 $(3.75 \mathrm{mcg} / \mathrm{mL}$ or $2 \mathrm{mcg} / \mathrm{kg}$ ) not ${ }^{(5)}$. Clonidine, alpha-2 receptor agonist, induce direct peripheral effects on action potentials or nerve fibres, can be added as adjuvant to LA. It decreases onset time, decresases intraoperative LA and analgesic requirement ${ }^{(5,9,14)}$. Fentanyl (50 mcg) accepted as an analgesic adjuvant to LA. Increase the quality and duration of the cervical block, decrease sedation requirement during CEA and delya the first analgesic requirement after operation ${ }^{(14)}$. Sindjelic et al. combined $10 \mathrm{~mL}$ bupivacaine $0.5 \%$ and $4 \mathrm{~mL}$ lidocaine $2 \%$ with the addition of either fentanyl $1 \mathrm{~mL}$ or saline placebo for deep cervical plexus block. They reported that addition of fentanyl to LA mixture enhanced the quality and duration of $\mathrm{CPB}^{(49)}$.

\section{Conclusion}

There is no consensus on anaesthetic choice for CEA. At present RA versus GA for patients undergoing CEA is still debate topic. Improvements in medical theraphy, use of cerebral monitoring, better timing for surgery after ischemic events, better surgical techniques, increased use of ultrasound for regional anaesthesia improve procedural outcomes. Despite all, anaesthesia method should be decided by considering the personal preferences of the surgeons and anesthesiologists, the patient's satisfaction and the conditions in the hospitals where we work.

\section{Acknowledgements}

I sincerely thank for Kadir Koray BAŞ, Health Science University İzmir Bozyaka Training and Research Hospital, Clinic of General Surgery, for his guidance and encouragement in carrying out this review. I thank İbrahim Erdinç, Health Science University İzmir Bozyaka Training and Research Hospital, Clinic of Cardiovascular Surgery, for his comments on the manuscript. 


\section{Ethics}

\section{Peer-review: Externally and internally peer-reviewed.}

Financial Disclosure: The author declared that this study received no financial support.

\section{References}

1. Patelis N, Diakomi M, Maskanakis A, Maltezos K, Schizas D, Papaioannou M. General versus local anesthesia for carotid endarterectomy: Special considerations. Saudi J Anaesth 2018;12:612-7.

2. Kavaklı AS, Ayoğlu RU, Öztürk NK, et al. Simultaneous Bilateral Carotid Endarterectomy under Cervical Plexus Blockade. Turk J Anaesthesiol Reanim 2015;43:367-70.

3. Zdrehuş C. Anaesthesia for carotid endarterectomy - general or locoregional? Rom J Anaesth Intensive Care 2015;22:17-24.

4. Kfoury E, Dort J, Trickey A, et al. Carotid endarterectomy under local and/ or regional anesthesia has less risk of myocardial infarction compared to general anesthesia: An analysis of national surgical quality improvement program database. Vascular 2015;23:113-9.

5. Guay J. Regional anesthesia for carotid surgery. Curr Opin Anaesthesiol 2008; 21:638-44.

6. Radak D, de Waard D, Halliday A, Neskovic M, Tanaskovic S. Carotid endarterectomy has significantly lower risk in the last two decades: should the guidelines now be updated? J Cardiovasc Surg 2018;59:586-99.

7. Licker M. Regional or general anaesthesia for carotid endarterectomy: Does it matter? Eur J Anaesthesiol 2016;33:241-43.

8. Pasin L, Nardelli P, Landoni G, et al. Examination of regional anesthesia for carotid endarterectomy. J Vasc Surg 2015;62:631-4.e1 [http://dx.doi. org/10.1016/j.jvs.2015.03.074].

9. Ciccozzi A, Angeletti C, Guetti C, et al. Regional anaesthesia techniques for carotid surgery: the state of art. J Ultrasound 2014;17:175-83.

10. Samanta S, Samanta S, Panda N, Haldar R. A unique anesthesia approach for carotid endarterectomy: Combination of general and regional anesthesia. Saudi J Anaesth 2014;8:290-3.

11. Casutt M, Breitenmoser I, Werner L, Seelos R, Konrad C. Ultrasoundguided carotid sheath block for carotid endarterectomy: a case series of the spread of injectate. Heart Lung and Vessel 2015;7:168-76.

12. Leblanc I, Chterev V, Rekik M, et al. Safety and efficiency of ultrasoundguided intermediate cervical plexus block for carotid surgery. Anaesth Crit Care Pain Med 2015;35:109-14.

13. Bhattathiri PS, Ramakrishnan Y, Vivar RA, et al. Effect of awake Carotid Endarterectomy under local anaesthesia on peri-operative blood pressure: blood pressure is normalised when carotid stenosis is treated under local anaesthesia. Acta Neurochir (Wien) 2005;147:839-45.

14. Stoneham MD, Stamou D, Mason J. Regional anaesthesia for carotid endarterectomy. Br J Anaesth 2015;114:372-83.

15. Seidel R, Zukowski K, Wree A, Schulze M. Ultrasound-guided intermediate cervical plexus and additional peripheral facial nerve block for carotid endarterectomy. A prospective pilot study. Anaesthesist 2018;67:907-13.
16. Koköfer A, Nawratil J, Felder TK, Stundner O, Mader N, Gerner P. Ropivacaine $0.375 \%$ vs. $0.75 \%$ with prilocaine for intermediate cervical plexus block for carotid endarterectomy A randomised trial. Eur J Anaesthesiol 2015;32:781-89.

17. Rössel T, Kerstring S, Heller AR, Koch T. Combination of high-resolution ultrasound-guided perivascular regional anesthesia of the internal carotid artery and intermediate cervical plexus block for carotid surgery. Ultrasound in Med Biol 2013;39:981-86.

18. Malik OS, Brovman EY, Urman RD. The Use of Regional or Local Anesthesia for Carotid Endarterectomies May Reduce Blood Loss and Pulmonary Complications. J Cardiothorac VascAnesth 2019;33:935-42.

19. GALA Trial Collaborative Group, Lewis SC, Warlow CP, et al. General anaesthesia versus local anaesthesia for carotid surgery (GALA): a multicentre, randomised controlled trial. Lancet 2008;372:2132-42.

20. Moulakakis GM, Avgerinos ED, Liapis CD. The hypothesis regarding the benefit of carotid endarterectomy under locoregional anesthesia in prevention of stroke may be unanswered. Angiology 2010;61:624-6.

21. Markovic D, Vlajkovic G, Sindjelic R, Markovic D, Ladjevic N, Kalezic N. Cervical plexus block versus general anesthesia in carotid surgery: single center experience. Arch Med Sci 2012;8:1035-40.

22. Lobo M, Mourão J, Afonso G. Carotid endarterectomy: review of 10 years of practice of general and locoregional anesthesia in a tertiary care hospital in Portugal. Braz J Anestesiol 2015;65:249-54.

23. Piffaretti G, Tarallo A, Franchin M, et al. Outcome Analysis of Carotid Cross-Clamp Intolerance during Carotid Endarterectomy under Locoregional Anesthesia. Ann Vasc Surg 2017;43:249-57.

24. Dellaretti M, de Vasconcelos LT, Dourado J, de Souza RF, Fontoura RR, de Sousa AA. Locoregional anesthesia for carotid endarterectomy: Identification of patients with intolerance to cross-clamping. World Neurosurg 2016;87:61-64.

25. Hye RJ, Voeks JH, Malas MB, et al. Anesthetic type and risk of myocardial infarction after carotid endarterectomy in the Carotid Revascularization Endarterectomy versus Stenting Trial (CREST). J Vasc Surg 2016;64:3-8. e1.

26. Hoefer J, Pierer E, Rantner B, et al. Ultrasound-guided regional anesthesia for carotid endarterectomy induces early hemodynamic and stress hormone changes. J Vasc Surg 2015;62:57-67.

27. Lomivorotov VV, Shmyrev VA, Moroz GB. Volatile Anesthesia for Carotid Endarterectomy: Friend or Foe for the Brain? J Cardiothorac Vasc Anesth 2018;32:1709-10.

28. Lee J, Huh U, Song S, Chung SW, Sung SM, Cho HJ. Regional Anesthesia with Dexmedetomidine Infusion: A Feasible Method for the Awake Test during Carotid Endarterectomy. Ann Vasc Dis 2016;9:295-99.

29. Tsujikawa S, Ikeshita K. Low-dose dexmedetomidine provides hemodynamics stabilization during emergence and recovery from general anesthesia in patients undergoing carotid endarterectomy: a randomized double-blind, placebo-controlled trial. J Anesth 2019;33:266-72.

30. Sideso E, Walton J, Handa A. General or local anesthesia for carotid endarterectomy--the "real-world" experience. Angiology 2011;62:609-13.

31. Kuzkov VV, Obraztsov MY, Ivashchenko OY, Ivashchenko NY, Gorenkov VM, Kirov MY. Total Intravenous Versus Volatile Induction and Maintenance of Anesthesia in Elective Carotid Endarterectomy: Effects 
on Cerebral Oxygenation and Cognitive Functions. J Cardiothorac Vasc Anesth 2018;32:1701-8.

32. Wang Q, Li YH, Wang TL, Feng H, Cai B. Protective Effect of Low-dose Sevoflurane Inhalation and Propofol Anesthesia on the Myocardium after Carotid Endarterectomy: A Randomized Controlled Trial. Chin Med J 2015;128:1862-6.

33. Luchetti M, Canella M, Zoppi M, Massei R. Comparison of regional anesthesia versus combined regional and general anesthesia for elective carotid endarterectomy: a small exploratory study. Reg Anesth Pain Med 2008;33:340-45.

34. Pandit JJ, Satya-Krishna S, Gration P. Superficial or deep cervical plexus block for carotid endarterectomy: a systematic review of complications. $\mathrm{Br}$ J Anaesth 2007;99:159-69.

35. Cedergreen P, Swiatek F, Nielsen HB. Local anaesthesia for carotid endarterectomy: Pro: protect the brain. Eur J Anaesthesiol 2016;33:236-37.

36. Mądro P, Dąbrowska A, Jarecki J, Garba P. Anaesthesia for carotid endarterectomy. Ultrasound-guided superficial/intermediate cervical plexus block combined with carotid sheath infiltration. Anaesthesiol Intensive Ther 2016;48:234-38.

37. Hakl M, Michalek P, Sevcík P, Pavlíková J, Stern M. Regional anaesthesia for carotid endarterectomy: an audit over 10 years. $\mathrm{Br} \mathrm{J}$ Anaesth 2007;99:415-20.

38. Kavaklı AS, Öztürk NK, Ayoğlu RU, et al. Comparison of Combined (Deep and Superficial) and Intermediate Cervical Plexus Block by Use of Ultrasound Guidance for Carotid Endarterectomy, J Cardiothorac Vasc Anesth 2016;30:317-22.

39. Yilmaz F, Bas K, Ulugolge B. A request for a clarification about classification and nomenclature of cervical plexus blocks. Ann Med Res 2019;26:966-7.

40. Seidel R, Zukowski K, Wree A, Schulze M. Ultrasound-guided intermediate cervical plexus block and perivascular local anesthetic infiltration for carotid endarterectomy : A randomized controlled trial. Anaesthesist 2016;65:917-24.

41. Martusevicius R, Swiatek F, Joergensen LG, Nielsen HB. Ultrasoundguided Locoregional Anaesthesia for Carotid Endarterectomy: A Prospective Observational Study. Eur J Vasc Endovasc Surg 2012;44:2730.

42. Kim JS, Sangwook Ko J, Bang S, Kim H, Lee SY. Cervical plexus block. Korean J Anesthesiol 2018;71:274-88.

43. Hariharan S, Naraynsingh V, Esack A, Ramdass MJ, Teelucksingh S, Naraynsingh A. Perioperative outcome of carotid endarterectomy with regional anesthesia: two decades of experience from the Caribbea. J Clin Anesth 2010;22:169-73.

44. Barone M, Diemunsch P, Baldassarre E, et al. Carotid Endarterectomy with Intermediate Cervical Plexus Block. Tex Heart Inst J 2010;37:297-300.

45. Gratz I, Deal E, Larijani GE, Domsky R, Goldberg ME. The number of injections does not influence absorption of bupivacaine after cervical plexus block for carotid endarterectomy. J Clin Anesth 2005;17:263-66.

46. Roessel T, Wiessner D, Heller AR, Zimmermann T, Koch T, Litz RJ. Highresolution ultrasound-guided high interscalene plexus block for carotid endarterectomy. Reg Anesth Pain Med 2007;32:247-53.

47. Ozturk NK, Kavakli AS, Sagdic K, Inanoglu K, Ayoglu U. A randomised controlled trial examining the effect on the addition of the mandibular block to cervical plexus block for carotid endarterectomy. J Cardiothorac Vasc Anesth 2018;32:877-82.

48. Cristallı A, Arlati S, Bettinelli L, Bracconaro G, Marconi G, Zerbi S. Regional anesthesia for carotid endarterectomy: a comparison between ropivacaine and levobupivacaine. Minerva Anestesiol 2009;75:231-7.

49. Sindjelic RP, Vlajkovic GP, Davidovic LB, Markovic DZ, Markovic MD. The addition of fentanyl to local anesthetics affects the quality and duration of cervical plexus block: a randomized, controlled trial. Anesth Analg 2010;111:234-37. 